\title{
Performance of Pulsating High-Frequency Current Injection Based Sensorless Control of PMSM
}

\author{
Wang Jianmin \\ School of Electrical Engineering \\ Shandong University \\ Jinan, China \\ e-mail:wjm@sdu.edu.cn
}

\begin{abstract}
In the paper the principle and performances of the pulsating current injection based sensorless control of permanent magnet synchronous motor (PMSM) are analyzed theoretically and investigated by simulations. In the analyses, the effects of the speed EMF terms and the deviation between the actual d-axis high-frequency current and the command, which results from the limited gain and bandwidth of the current control loop, are all taken into account. It is shown that the pulsating current injection method can achieve stable position estimation in a wide speed range. But appreciable position errors will result at high speeds due to the crosscoupling effects of the speed EMFs and the tracking error between the actual and command carrier current. In order to improve the performance, a modified scheme is proposed. Its validity is confirmed by simulations.
\end{abstract}

Keywords-permanent magnet synchronous motor; sensorless control; pulsating current injection; performance; effect of speed EMF

\section{INTRODUCTION}

In the control of PMSM, the rotor position and speed information are necessary, which are usually provided by a shaft mounted mechanical position sensor. In order to reduce the cost, size, and to improve reliability by eliminating the sensor and related cabling connection, the sensorless control technique emerged and has become an important research topic in recent years.

A variety of sensorless control techniques have been proposed. They can be classified as two main categories [1][3]: methods based on fundamental model and methods based on the spatial saliency of rotor. The fundamental model based methods depend on the fundamental excitation and the back-EMF of machine. Though they work successfully at medium and high rotor speeds, but fail at very low speed due to the small magnitude and low frequency of the back-EMF signal. The spatial saliency based methods are often referred to as signal injection methods, in which a second excitation signal is injected into the motor winding to track the saliency of the motor.

Among the signal injection based sensorless control of PMSM, the most attractive methods are the rotating highfrequency voltage injection method and the pulsating high- frequency voltage injection method. They can achieve accurate position estimation over a wide speed range, including zero speed, and are insusceptible to motor parameter variations [1]-[7]. So far, although the highfrequency current injection methods are often mentioned, but further researches are scarce.

Reference [8] presents a pulsating current injection scheme recently. But only the performances at low speed are studied under the hypotheses that the $\mathrm{d}$ - and q-axis carrier current in the estimated rotor frame are equal to the commands, and the speed EMF terms are negligible.

In this paper, the principle and performances of the pulsating high-frequency current injection based sensorless control of PMSM are analyzed theoretically and investigated by simulations. First, the expressions of the high-frequency voltage induced by the injected carrier current are derived when the speed EMF terms are taken into account. Then taking the deviation between the actual d-axis carrier current and the command into account, the position estimation error expression is deduced. The effects of the current controller on the position error are analyzed and investigated. Finally, in order to improve the position estimation accuracy, the modified scheme is proposed and its performances are investigated by simulations.

\section{Principle OF PUlsating CURRENT InJeCtion}

In the pulsating current injection method, a pulsating high-frequency sinusoidal current is superimposed onto the $\mathrm{d}$-axis of the estimated rotor frame, and the high-frequency voltage at the estimated q-axis is used to estimate the rotor position. The diagram is shown in Fig.1 [8], in which the qaxis carrier voltage is got from the output of the q-axis current regulator.

\section{A. Expressions of the Induced High-Frequency Voltage}

When the speed EMF terms are taken into account, the high frequency model of PMSM for the carrier signal only can be expressed as (1) in the rotor reference frame [7].

$$
\left[\begin{array}{c}
u_{d c}^{r} \\
u_{q c}^{r}
\end{array}\right]=\left[\begin{array}{cc}
R_{s}+L_{d} p & -\omega_{r} L_{q} \\
\omega_{r} L_{d} & R_{s}+L_{q} p
\end{array}\right]\left[\begin{array}{l}
i_{d c}^{r} \\
i_{q c}^{r}
\end{array}\right]
$$




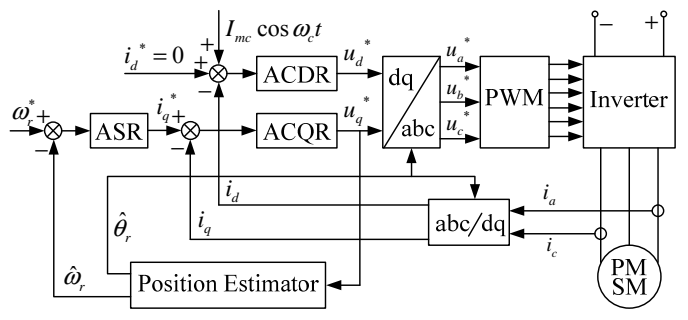

Figure 1. Diagram of the pulsating current injection based sensorless control of PMSM

Where $u_{d c}^{r}, u_{q c}^{r}, i_{d c}^{r}, i_{q c}^{r}, L_{d}, L_{q}$ are respectively the dand q-axis high-frequency voltage, current, and inductance; $R_{s}$ is the stator resistance; $\omega_{r}$ is the electrical angular speed of the rotor; and $p$ is the differential operator.

Convert (1) into the estimated rotor frame by using transformation matrix (2), (3) can be obtained in steady state.

$$
\begin{aligned}
& C_{r / \hat{r}}=\left[\begin{array}{cc}
\cos \Delta \theta_{r} & -\sin \Delta \theta_{r} \\
\sin \Delta \theta_{r} & \cos \Delta \theta_{r}
\end{array}\right] \\
& {\left[\begin{array}{l}
u_{d c}^{\hat{r}} \\
u_{q c}^{\hat{r}}
\end{array}\right]=\left[\begin{array}{cc}
L_{0}-\Delta L \cos 2 \Delta \theta_{r} & -\Delta L \sin 2 \Delta \theta_{r} \\
-\Delta L \sin 2 \Delta \theta_{r} & L_{0}+\Delta L \cos 2 \Delta \theta_{r}
\end{array}\right] \cdot \frac{\mathrm{d}}{\mathrm{d} t}\left[\begin{array}{c}
i_{d c}^{\hat{r}} \\
i_{q c}^{\hat{r}}
\end{array}\right]} \\
& +\left[\begin{array}{cc}
R_{s}+\omega_{r} \Delta L \sin 2 \Delta \theta_{r} & -\omega_{r}\left(L_{0}+\Delta L \cos 2 \Delta \theta_{r}\right) \\
\omega_{r}\left(L_{0}-\Delta L \cos 2 \Delta \theta_{r}\right) & R_{s}-\omega_{r} \Delta L \sin 2 \Delta \theta_{r}
\end{array}\right]\left[\begin{array}{l}
i_{d c}^{\hat{r}} \\
i_{q c}^{\hat{r}}
\end{array}\right]
\end{aligned}
$$

Where, $\Delta \theta_{r}=\theta_{r}-\hat{\theta}_{r}, \theta_{r}$ and $\hat{\theta}_{r}$ are the actual and estimated rotor position; $u_{d c}^{\hat{r}}, u_{q c}^{\hat{r}}, i_{d c}^{\hat{r}}, i_{q c}^{\hat{r}}$ are the d- and qaxis carrier voltage and current in the estimated rotor frame.

$$
L_{0}=\left(L_{d}+L_{q}\right) / 2, \Delta L=\left(L_{q}-L_{d}\right) / 2
$$

From Fig.1, the commands of the injected carrier current are as follows: $i_{d c}^{\hat{r}^{*}}=I_{m c} \cos \omega_{c} t, i_{q c}^{\hat{r}^{*}}=0$, Where $I_{m c}$ is the amplitude of the carrier current. Assume the current controllers are ideal, the actual carrier currents will be equal to the commands, i.e.

$$
\left[\begin{array}{l}
i_{d c}^{\hat{r}} \\
i_{q c}^{\hat{r}}
\end{array}\right]=\left[\begin{array}{l}
i_{d c}^{\hat{r}^{*}} \\
i_{q c}^{\hat{r}^{*}}
\end{array}\right]=\left[\begin{array}{c}
I_{m c} \cos \omega_{c} t \\
0
\end{array}\right] .
$$

Substituting (5) into (3), the d- and q-axis carrier voltages are obtained and expressed as (6) and (7) respectively.

$$
\begin{aligned}
u_{d c}^{\hat{r}}= & -\omega_{c}\left(L_{0}-\Delta L \cos 2 \Delta \theta_{r}\right) I_{m c} \sin \omega_{c} t \\
& +\left(R_{s}+\omega_{r} \Delta L \sin 2 \Delta \theta_{r}\right) I_{m c} \cos \omega_{c} t
\end{aligned}
$$

$$
\begin{aligned}
u_{q c}^{\hat{r}} & =\omega_{c} \Delta L I_{m c} \sin 2 \Delta \theta_{r} \sin \omega_{c} t \\
& +\omega_{r}\left(L_{0}-\Delta L \cos 2 \Delta \theta_{r}\right) I_{m c} \cos \omega_{c} t
\end{aligned}
$$

\section{B. Position and Speed Estimation}

The rotor position and speed can be estimated from $u_{q c}^{\hat{r}}$, the process is shown in Fig. 2 .

According to Fig.2, the tracking error signal $\varepsilon$, which is the input of the speed estimator, can be expressed as (8).

$$
\varepsilon=L P F\left(u_{q c}^{\hat{r}} \times 2 \sin \omega_{c} t\right)=\omega_{c} \Delta L I_{m c} \sin 2 \Delta \theta_{r}
$$

It is seen clearly that when the signal $\varepsilon$ is regulated to zero, $\Delta \theta_{r}$ will be zero. This means that no position error will result, even though the speed EMF terms are taken into account and a nonzero q-axis carrier voltage is produced as shown in (7). But unfortunately, this conclusion is obtained under the ideal current controller condition. In fact, position estimation errors will be resulted from the real (non-ideal) current regulators. This will be discussed in the next section.

\section{EFFECT OF CURRENT CONTROLLER ON POSITION ESTIMATION ERROR}

\section{A. Position Error Resulting from the Current Deviation}

Suppose that the amplitude of the actual d-axis carrier current is $I_{m c}^{\prime}$, the phase lags the command a $\varphi$ angle, and the q-axis carrier current is regulated perfectly. Then the actual injected carrier current can be expressed as (9).

$$
\left[\begin{array}{l}
i_{d c}^{\hat{r}} \\
i_{q c}^{\hat{r}}
\end{array}\right]=\left[\begin{array}{c}
I_{m c}^{\prime} \cos \left(\omega_{c} t-\varphi\right) \\
0
\end{array}\right]
$$

Substituting (9) into (3), the q-axis high-frequency voltage is obtained as (10).

$$
\begin{aligned}
u_{q c}^{\hat{r}} & =\omega_{c} \Delta L I_{m c}^{\prime} \sin 2 \Delta \theta_{r} \sin \left(\omega_{c} t-\varphi\right) \\
& +\omega_{r}\left(L_{0}-\Delta L \cos 2 \Delta \theta_{r}\right) I_{m c}^{\prime} \cos \left(\omega_{c} t-\varphi\right)
\end{aligned}
$$

According to Fig.2, the signal $\varepsilon$ is derived as (11).

$$
\begin{aligned}
\varepsilon & =\omega_{c} \Delta L I_{m c}^{\prime} \sin 2 \Delta \theta_{r} \cos \varphi \\
& +\omega_{r}\left(L_{0}-\Delta L \cos 2 \Delta \theta_{r}\right) I_{m c}^{\prime} \sin \varphi
\end{aligned}
$$

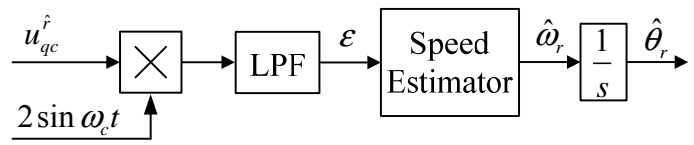

Figure 2. Rotor position and speed estimation in the pulsating current injection method 
Let $\mathcal{\varepsilon}=0$, the position error can be obtained as (12).

$$
\begin{aligned}
\Delta \theta_{r} & =\frac{1}{2} \arcsin \left(\frac{-\omega_{r} L_{0}+\omega_{r} \Delta L \cos 2 \Delta \theta_{r}}{\omega_{c} \Delta L} \tan \varphi\right) \\
& \approx-\frac{1}{2} \arcsin \left[\frac{2 \omega_{r} L_{d}}{\omega_{c}\left(L_{q}-L_{d}\right)} \tan \varphi\right] \\
& \approx-\frac{\omega_{r} L_{d}}{\omega_{c}\left(L_{q}-L_{d}\right)} \varphi
\end{aligned}
$$

In the following analyses and investigation, assume that the classical PI regulators are used. The transfer functions of the d-axis current regulator and the d-axis carrier voltage to the d-axis carrier current can be immediately expressed as (13) and (14) respectively.

$$
\begin{gathered}
\frac{U_{d c}(s)}{I_{d c}^{*}(s)-I_{d c}(s)}=K_{p}\left(1+\frac{1}{T_{r} s}\right) \\
\frac{I_{d c}(s)}{U_{d c}(s)}=\frac{1}{R_{s}+L_{d} s}
\end{gathered}
$$

Where $K_{p}$ is the proportional gain, and $T_{r}$ is the integral time constant.

If the classical pole/zero cancellation methodology is used in the design, the close-loop transfer function can be obtained as (15). Thus the lagging phase angle $\varphi$ of the actual d-axis carrier current can be expressed as (16).

$$
\begin{gathered}
\frac{I_{d c}(s)}{I_{d c}{ }^{*}(s)}=\frac{1}{1+s / \omega_{d}} \\
\varphi=\arctan \left(\frac{\omega_{c}}{\omega_{d}}\right)
\end{gathered}
$$

Where, $\omega_{d}=K_{p} / L_{d}$, is the cut-off frequency of the d-axis current control loop.

Substituting (16) into (12), the position error can be reexpressed as (17).

$$
\Delta \theta_{r} \approx-\frac{1}{2} \arcsin \left[\frac{2 \omega_{r} L_{d}}{\omega_{d}\left(L_{q}-L_{d}\right)}\right] \approx-\frac{\omega_{r} L_{d}}{\omega_{d}\left(L_{q}-L_{d}\right)}
$$

\section{B. Simulation Investigations}

Simulation investigations are carried out with two motors whose $L_{q} / L_{d}$ values are 3.03 and 1.5 respectively. Motor parameters are given in Table I. The amplitude and frequency of the injected d-axis carrier current are $0.1 \mathrm{~A}$ and $500 \mathrm{~Hz}$.

Fig.3 and Fig.4 compare the simulated and calculated position estimation errors of motor I and motor II respectively, here the cut-off frequencies of the $\mathrm{d}$ - and q-axis

TABLE I. MOTOR PARAMETERS

\begin{tabular}{|l|c|c|}
\hline \multicolumn{1}{|c|}{ Motor } & I & II \\
\hline Stator resistance $R_{s}(\Omega)$ & 2.2 & 2.875 \\
\hline d-axis inductance $L_{d}(\mathrm{mH})$ & 6.5 & 8.5 \\
\hline q-axis inductance $L_{q}(\mathrm{mH})$ & 19.69 & 12.75 \\
\hline Permanent magnet flux $\psi_{f}(\mathrm{~Wb})$ & 0.243 & 0.175 \\
\hline Moment of inertia $J\left(\mathrm{gm}^{2}\right)$ & 0.08 & 0.8 \\
\hline Number of pole pairs $n_{p}$ & 1 & 4 \\
\hline
\end{tabular}

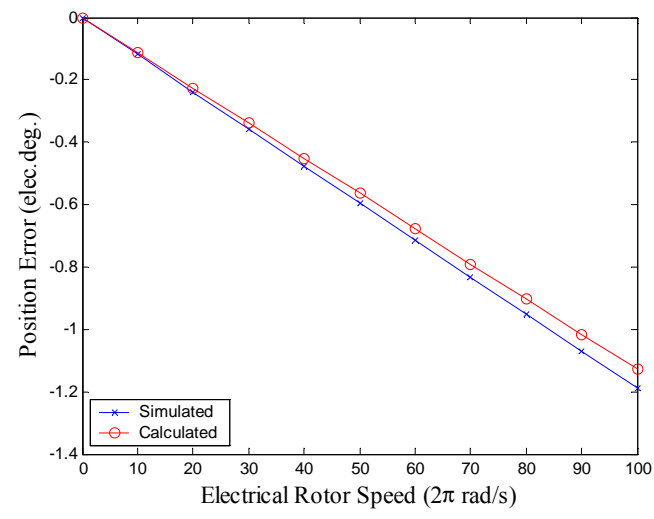

Figure 3. Position estimation errors of the motor I

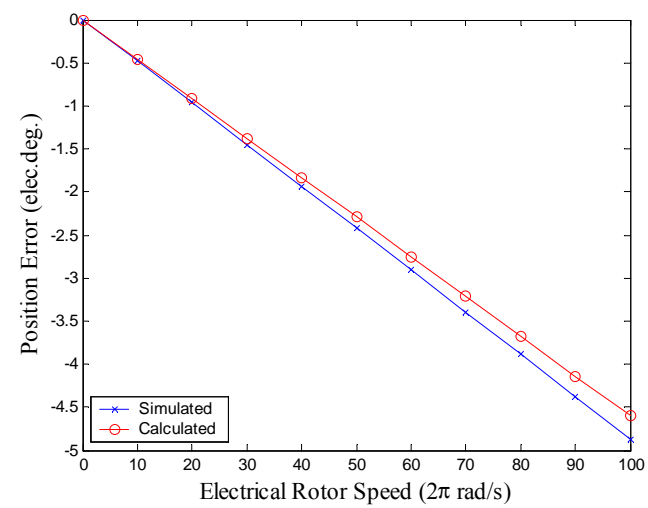

Figure 4.Position estimation errors of the motor II

current control loop are $\omega_{d}=5 \omega_{c}$, and $\omega_{q}=100 \omega_{c}$. It is seen clearly that the calculated results coincide well with the simulated ones, and the position errors of motor II are obviously larger than those of the motor I due to its low saliency.

It should be pointed out that: the position error formula (12) and (17) are accurate enough only when the amplitude of $i_{q c}^{\hat{r}}$ is small enough (very near zero), because they are derived under the condition that the q-axis carrier current is perfectly regulated, i.e. $i_{q c}^{\hat{r}}=0$. But the actual current can not be zero with a PI controller, and it should be suppressed 
as much as possible by high PI controller gain. This is why the $\omega_{q}$ is designed so large in the simulations.

\section{MOdified PUlsating CURRENT INJECTION METHODS}

In order to improve the position estimation accuracy of the pulsating current injection method, a modified scheme is proposed and investigated by simulations in this section.

\section{A. Modified Scheme with Improved Position Estimator}

In the modified scheme, the position estimator shown in Fig. 2 is modified and shown in Fig.5. The only difference between them is that the demodulation signal $2 \sin \omega_{\mathrm{c}} t$ in Fig. 2 is replaced by $2 \sin \left(\omega_{c} t-\varphi\right)$, thus the phase lagging of the d-axis carrier current resulted from the current regulator is taken into account in the position estimation process.

According to Fig.5, in the modified scheme the tracking error signal $\varepsilon$ is expressed as (18).

$$
\varepsilon=L P F\left[u_{q c}^{\hat{r}} \times 2 \sin \left(\omega_{c} t-\varphi\right)\right]
$$
(19).

Substituting (10) into (18), the signal $\varepsilon$ can be obtained as

$$
\varepsilon=\omega_{c} \Delta L I_{m c}^{\prime} \sin 2 \Delta \theta_{r}
$$

When $\varepsilon$ is regulated to zero, $\Delta \theta_{r}$ will equal zero, thus zero position error is theoretically achieved in steady state.

\section{B. Performances and Discussions}

The performances of the modified scheme are investigated by simulations with the motor II under different operation conditions. Some representative results at $\omega_{r}=200 \pi$ $\mathrm{rad} / \mathrm{s}$ are shown in Table II. It is seen clearly that the modified scheme can achieve accurate position estimations, and is insensitive to motor parameter variations.

In addition, it is noted that the stator resistance has little effect on the position errors; if the d-axis inductance increases or the q-axis inductance decreases, i.e. the saliency ratio of the motor become low, relatively larger position errors will result.

\section{CONCLUSIONS}

In the paper, the principle of the pulsating current injection based sensorless control of PMSM and its performances in a wide speed range are analyzed theoretically and investigated by simulations. Especially, the effects of the speed EMF and the non-ideal characteristics of the current controller are taken into account in the analyses. In order to improve the performance, the modified scheme is proposed. The main conclusions can be summarized as follows:

The pulsating current injection method can achieve stable position estimations in a wide speed range, but appreciable position errors will result at high speeds, especially for the motor with low saliency ratio. This is attributed to the cross-

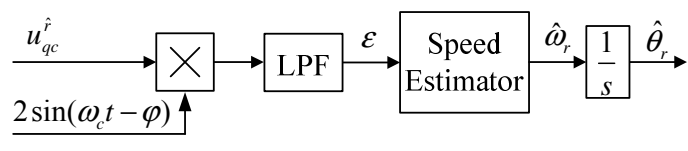

Figure 5. Improved position estimator

\section{TABLE II. POSITION ERRORS IN DIFFERENT CONDITIONS}

\begin{tabular}{|c|c|c|c|}
\hline \multicolumn{2}{|c|}{ Parameters } & Original & Modified \\
\hline \multicolumn{2}{|c|}{ Matched } & -4.882 & -0.2315 \\
\hline \multirow{2}{*}{$R_{s}$} & $+50 \%$ & -4.860 & -0.2113 \\
\cline { 2 - 4 } & $-50 \%$ & -4.906 & -0.2533 \\
\hline \multirow{2}{*}{$L_{d}$} & $+20 \%$ & -12.133 & -2.247 \\
\cline { 2 - 4 } & $-20 \%$ & -2.235 & -0.3783 \\
\hline \multirow{2}{*}{$L_{q}$} & $+20 \%$ & -3.065 & -0.1730 \\
\cline { 2 - 4 } & $-20 \%$ & -12.562 & -0.4657 \\
\hline
\end{tabular}

coupling effects of the speed EMF and the deviation between the actual carrier current and the command resulting from the current controllers.

The proposed scheme is very effective in compensating the d-axis carrier current deviation resulting from the d-axis current regulator. When the cut-off frequency of the q-axis current control loop is high enough, the modified scheme can achieve very good performances even if in the case of mismatched motor parameters. But the effects and compensation of the q-axis carrier current are still not taken into account in the modified scheme. This work is currently carrying out.

\section{REFERENCES}

[1] J. Holtz, "Developments in sensorless AC drive technology," Prco. IEEE on Power Electronics and Drives Systems, pp. 9-16, 2005.

[2] R. D. Lorenz, "Practical issues and research opportunities when implementing zero speed sensorless control," Proc. ICEMS2001, pp. $1-10$, August 2001.

[3] S. Kim, and S. K. Sul, "Sensorless control of AC motor-Where are we now," Proc. ICEMS2011, August 2011.

[4] P. L. Jansen, and R. D. Lorenz, "Transducerless position and velocity estimation in induction and salient AC machines," IEEE Trans. on Ind. Appl., vol. 31, pp. 240-247, March/April 1995.

[5] M. J. Corley, and R. D. Lorenz, "Rotor position and velocity estimation for a permanent magnet synchronous machine at standstill and high speeds," Proc. IEEE IAS Annual Meeting, pp. 36-41, Oct. 1996.

[6] Wang Jianmin, and Gao Jianwei, "Analysis of position estimation error resulted from filter in carrier signal injection based sensorless control of PMSM," Proc. ICEMS2011, August 2011.

[7] Wang Jianmin, and Gao Jianwei, "Influence of speed EMF on position estimation error in carrier signal injection based sensorless control of PMSM,” Proc. ICEMS2012, October 2012.

[8] Liu Ying, Zhou Bo, Feng Ying, Wei Jiadan, and Shi Mingming, "Sensorless control with two types of pulsating high frequency signal injection methods for SPMSM at low speed," Proc. PESA2011, June 2011 . 